\title{
The Participatory Design of an Adaptive Interface to Support Users with Changing Pointing Ability
} \author{
Aqueasha
}

\author{
${ }^{2}$ Information Systems Department \\ University of Maryland, \\ Baltimore County \\ Baltimore, MD, USA \\ \{foadhamidi, tejasb1, cat19, \\ amyhurst\}@umbc.edu
}

Catherine Hornback ${ }^{2}$, Casey Means ${ }^{4}$, Amy Hurst ${ }^{2}$

\author{
${ }^{3}$ DUB Group \\ Information School \\ University of Washington \\ Seattle, WA, USA \\ xyleques@uw.edu
}

\author{
${ }^{4}$ Department of \\ Computer Science \\ Rhodes College \\ Memphis, TN, USA \\ meaac-16@rhodes.edu
}

\begin{abstract}
Individuals who experience temporary, intermittent, or gradual changes in pointing ability may encounter frustrating experiences when using computer input devices. Personalized pointing systems that automatically assess changes in performance and provide individualized information and assistance may benefit these users. However, there has been little inquiry into this populations' expectations for interacting with these types of systems.

We describe a participatory design process in which we used a technology probe to assess the information needs and expectations of 27 individuals who experience occasional changes in pointing ability, through interactions with and discussion regarding a highfidelity personalized pointing prototype. Participants preferred notification and adaptation interactions that provided them with control and explanation of system actions, instead of abstract notifications and automatic adaptations. We describe how we applied these finding in the design of the PINATA system.
\end{abstract}

\section{CCS Concepts}

Human-centered computing $\rightarrow$ Accessibility $\rightarrow$ Accessibility systems and tools

\section{Keywords}

Pointing problems; Web Navigation; Adaptive Systems

\section{INTRODUCTION AND BACKGROUND}

For individuals that experience temporary, intermittent, or gradual changes in pointing ability due to a physical impairment, environmental issue or age, using an input device to interact with user interface elements can be difficult. An individual with early stage Parkinson's disease may experience significant differences in his or her ability to control the mouse depending on medication regimen or time of day [4]. Alternatively, a younger adult's ability to control a mouse may change depending on his or her sleep schedule, excitement, or caffeine use [4]. An older adult user may experience changes in hand mobility due to the natural aging process [5]. These individuals may not have been diagnosed with motor impairment, and may not identify as assistive technology users. They may be unaware of changes, or if aware, may find it difficult to identify assistive technology solutions that can support

Permission to make digital or hard copies of part or all of this work for personal or classroom use is granted without fee provided that copies are not made or distributed for profit or commercial advantage and that copies bear this notice and the full citation on the first page. Copyrights for third-party components of this work must be honored. For all other uses, contact the Owner/Author.

ASSETS '17, October 29-November 1, 2017, Baltimore, MD, USA

C 2017 Copyright is held by the owner/author(s).

ACM ISBN 978-1-4503-4926-0/17/10.

https://doi.org/10.1145/3132525.3134810 their dynamically changing needs, leading to frustrating computer experiences and further impeding their computer use $[1,4,6]$.

Systems that provide personalized (or adaptive) pointing support are a promising solution for individuals that experience changes in pointing ability because they can adapt to user behavior and provide real-time assistance tailored to immediate needs $[6,7]$. However, researchers have yet to identify the information needs and expectations when interacting with these systems.

\section{INCLUDING USERS IN DESIGN}

To include participants in the design process of PINATA as early as possible, we developed and used a prototype of the system as a design probe [2]. We used the prototype to demonstrate different forms of notifications, mechanisms to inform the user about their pointing performance, and assistance activation modes, mechanisms to activate interface modification to assist with pointing tasks (e.g., zooming in the screen). Figure 1 shows the 3 notification designs that were shown to the participants.

We recruited 27 participants with diverse abilities: 12 younger adults (18 - 34 years), 10 older adults (ages 65 years or older), and 5 adults (ages 55 years or older) with early-stage Parkinson's. No participants reported impairments that would completely impede their use of a computer; 23 participants reported experiencing intermittent difficulty when using a pointing device.

We engaged participants in interview sessions where we demonstrated each of the notifications and had them interact with the prototype. We asked the participants about their preferences with respect to notification visibility, information delivery, information needs, and assistance information and activation preference. After collecting the data from our users, we conducted a thematic analysis and identified three themes to inform the system's design.

\section{DESIGN RECOMMENDATIONS AND IMPLEMENTATIONS}

After collecting the data from our users, we conducted a thematic analysis and identified three themes to inform the system's design. In the following subsections, we present the three main themes that emerged and how we incorporated them into the prototype.

\subsection{Put the User in Control}

Most participants (78\%) preferred a system that combined automated assistance with explicit user input. The participants wanted to be able to control the type of errors that were detected and how the system would respond. One participant provided the example of "predictive text" when typing and mentioned that automatic assistance can be annoying when it suggests words other than what they were trying to type. 


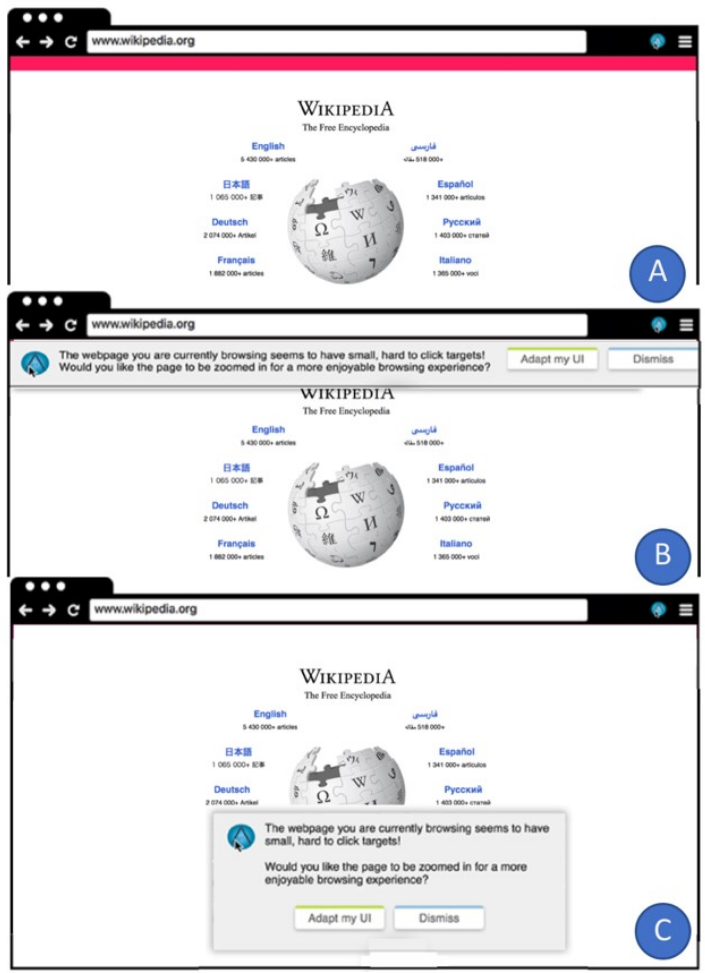

Figure 1. The Bar notification (A) consists of a horizontal bar. The Bar+ notification (B) includes text and interactive buttons. The Dialog box (C) is a popup alert with text and buttons.

To implement this preference, we provide users with a settings window (see Figure 2) that allows them (and their caregivers) to tweak different system settings, including the type of detected errors and the frequency of notifications provided. Additionally, the system allows the users to download and view all the data PINATA collects to automatically detect errors.

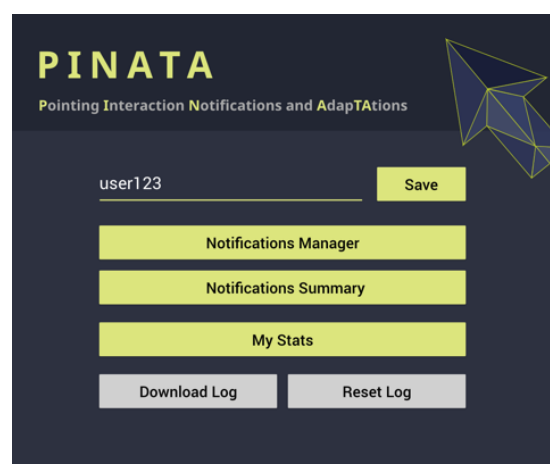

Figure 2. PINATA allows each user to review and specify their preferences.

\subsection{Keep the User Informed}

Participants did not like the Bar Notification because it did not explain why a notification was given. Instead, participants preferred the dialog box $(63 \%)$ or Bar+ $(30 \%)$ notifications because they provided this information and obvious support. This result is in accord with previous recommendations (e.g., [3]).

We incorporated this preference into PINATA's current design by providing textual information about why a notification is provided, with an option to see more information about it. For example, if a user had difficulty clicking on links, a message would state, "In the last 10 minutes, $75 \%$ of links have been too small to click. Would you like to zoom in on the page?". The user can then choose to deploy adaptation, get more information or ignore the message.

\subsection{Help the User Trust the System}

Most of our participants were confident that a computer could provide accurate automatic adaptations $(85 \%)$ and provide them quickly $(70 \%)$. However, many users $(78 \%)$ were concerned about trusting that the system could act in accordance to their needs and preferred an activation mode that required their confirmation.

We implemented a mixed-initiative approach to activating assistance, where the user can confirm and activate the assistance when it is suggested by the system. Once the user is familiar with system, they have the choice to automate the assistance activation.

\section{CONCLUSION}

We investigated how individuals who experience intermittent or gradual declines in hand mobility want to be notified about pointing changes and their preference for assistance activation. We learned that users wanted systems that could 1) build trust by acting in a way that met individual user expectations, 2) keep them informed, and 3) put them in control of decision-making. We described how these preferences were incorporated into our PINATA system.

\section{ACKNOWLEDGMENTS}

This project was funded by a NIDILRR grant \#-90DP0061-01-00.

\section{REFERENCES}

[1] Gregor, P., Newell, A. and Zajicek, M. 2002. Designing for dynamic diversity: interfaces for older people. In Proc. ASSETS'02, 151-156.

[2] Gaver, B., Dunne, T., and Pacenti, E. 1999. Design: cultural probes. Interactions, 6(1), 21-29.

[3] Kulesza, T., Stumpf, S., Burnett, M. and Kwan, I. 2012. Tell me more?: the effects of mental model soundness on personalizing an intelligent agent. In Proc. of CHI '12, 1-10.

[4] Moffatt, K. and McGrenere, J. 2009. Exploring Methods to Improve Pen-Based Menu Selection for Younger and Older Adults. Trans. Access. Comput. 2, 1, Article 3, 34 pages.

[5] Pak, R., and McLaughlin, A. C. 2010. Designing displays for older adults. Boca Raton, FL: CRC Press.

[6] Peissner, M., Häbe, D., Janssen, D. and Sellner, T. 2012. MyUI: generating accessible user interfaces from multimodal design patterns. In Proc. of EICS '12, 81-90.

[7] Rothrock, L., Koubek, R., Fuchs, F., Haas, M., and Salvendy, G. 2010. Review and reappraisal of adaptive interfaces: Toward biologically inspired paradigms. TIES, 3(1), 47-84. 